\title{
Application of $p$-regularity theory to nonlinear boundary value problems
}

Wiesław Grzegorczyk ${ }^{1 \dagger}$, Beata Medak ${ }^{1 *+}$ and Alexey A Tret'yakov ${ }^{1,2,3 \dagger}$

\section{${ }^{*}$ Correspondence:}

bmedak@uph.edu.p

'Siedlce University of Natural

Sciences and Humanities, 3-go Maja

54, Siedlce, 08-110, Poland

† Equal contributors

Full list of author information is

available at the end of the article

\begin{abstract}
The paper studies the question of solution existence to a nonlinear equation in the degenerate case. This question is studied for three particular boundary value problems for ordinary and partial second-order differential equations. The so-called $p$-regularity theory is applied to these purposes as an effective apparatus to investigate many nonlinear mathematical, physical and numerical problems. All results obtained in the paper are based on the constructions of this theory whose basic concepts were described by Tret'yakov. We recall the main definitions and theorems of $p$-regularity theory and illustrate the results by examples including singular boundary value problems. In the first and second ones, the description of solutions by a tangent cone at an initial point are given. In the third example, we formulate a sufficient condition for $p$-regularity $(p=2)$, which can be tested using the notion of resultant.

MSC: 47J05; 34B15; 34B16
\end{abstract}

Keywords: $p$-regularity; $p$-factor operator; $p$-kernel; singularity; differential equations; nonlinear boundary value problems

\section{Introduction}

In the paper, we study the question of solution existence to a nonlinear equation

$$
F(x)=0,
$$

where $X, Y$ are Banach spaces and $F \in \mathcal{C}^{p}(X, Y)(p \geq 2)$. Let $x_{*}$ be a solution to this equation, i.e., $F\left(x_{*}\right)=0$. The above problem is called regular at the point $x_{*}$ if $\operatorname{Im} F^{\prime}\left(x_{*}\right)=Y$. Otherwise, problem (1) is called irregular, degenerate or singular at $x_{*}$.

The construction of $p$-regularity [1-4] gives new possibilities for solving or describing degenerate problems (see, for instance, [5-7]). We are going to use it to certain questions that appear in many numerical applications. Namely, we consider the equation of rod bending and the nonlinear Laplace equation that describe many mathematical physics problems like string oscillation, membrane oscillation and so on.

The following degenerate nonlinear boundary value problem:

$$
F(x, \varepsilon)=x^{\prime \prime}+(1+\varepsilon)\left(x+x^{2}\right)=0
$$

where $F: \mathcal{C}_{0}^{2}[0, \pi] \times \mathbb{R} \rightarrow \mathcal{C}[0, \pi]$ and $\mathcal{C}_{0}^{2}[0, \pi]=\left\{x \in \mathcal{C}^{2}[0, \pi]: x(0)=x(\pi)=0\right\}$, is the first of them.

@2013 Grzegorczyk et al.; licensee Springer. This is an Open Access article distributed under the terms of the Creative Commons Attribution License (http://creativecommons.org/licenses/by/2.0), which permits unrestricted use, distribution, and reproduction in any medium, provided the original work is properly cited. 
The second one is a modification of equation (2), i.e.,

$$
F(x, \varepsilon)=x^{\prime \prime}+x+\varepsilon x^{p-1}+x^{p}=0,
$$

where $F: \mathcal{C}_{0}^{2}[0, \pi] \times \mathbb{R} \rightarrow \mathcal{C}[0, \pi], p \geq 2$.

The third one is the partial differential equation of the form

$$
F(u, \varepsilon)=\Delta u-(\varepsilon-10) g(u)=0,
$$

where $F: \mathcal{C}_{0}^{2}[\Omega] \times \mathbb{R} \rightarrow \mathcal{C}[\Omega], \Omega=[0, \pi] \times[0, \pi], \Delta$ is the Laplacian and $\mathcal{C}_{0}^{2}[\Omega]=\{u \in$ $\left.\mathcal{C}^{2}[\Omega]:\left.u\right|_{\partial \Omega}=0\right\}$; moreover, $g: \Omega \rightarrow \mathbb{R}$ is a certain function such that $g(0)=0, g^{\prime}(0)=1$, $g^{\prime \prime}(0) \neq 0$.

Note that the numbers -1 and -10 are the eigenvalues of the operators $(\cdot)^{\prime \prime}$ and $\Delta$ in equations (2) and (4), respectively. Moreover, in the first example, we could take $g(x)$ instead of $x+x^{2}$ such that $g(0)=0, g^{\prime}(0)=1, g^{\prime \prime}(0) \neq 0$. It is important that the 2-regularity condition is fulfilled for the mapping $F(x, \varepsilon)$ at the point $(0,0)$. We chose equation (3) in order to expose our results not only for $p=2$. In equation (4) we have taken $\lambda=-10$ as an arbitrary representative element of eigenvalues of the Laplacian of the form $-\left(k^{2}+n^{2}\right)$, $k, n \in \mathbb{N}$. Of course it is possible to take for example $\lambda=-2$.

We begin with some notation. Suppose $X$ and $Y$ are Banach spaces and denote the space of all continuous linear operators from $X$ to $Y$ by $\mathcal{L}(X, Y)$. Let $p$ be a natural number, and let $B: X \times X \times \cdots \times X(p$-copies of $X) \rightarrow Y$ be a continuous symmetric $p$-multilinear mapping. The $p$ form associated to $B$ is the map $B[\cdot]^{p}: X \rightarrow Y$ defined by $B[x]^{p}=B(x, x, \ldots, x)$ for $x \in X$. Alternatively, we may simply view $B[\cdot]^{p}$ as a homogeneous polynomial $Q: X \rightarrow Y$ of degree $p$, and hence $Q(\alpha x)=\alpha^{p} Q(x)$. Throughout this paper, we assume that the mapping $F: X \rightarrow Y$ is $p$-times continuously Fréchet differentiable on $X$ and its $p$ th order derivative at $x \in X$ will be denoted as $F^{(p)}(x)$ (a symmetric multilinear map of $p$ copies of $X$ to $Y$ ) and the associated $p$-form, also called a $p$ th-order mapping, is

$$
F^{(p)}(x)[h]^{p}=F^{(p)}(x)[h, h, \ldots, h] .
$$

We also use the notation

$$
\operatorname{Ker}^{p} F^{(p)}(x)=\left\{h \in X: F^{(p)}(x)[h]^{p}=0\right\}
$$

and refer to it as the $p$-kernel of the $p$ th-order mapping.

The set $M\left(x_{*}\right)=\left\{x \in X: F(x)=F\left(x_{*}\right)=0\right\}$ is called the solution set for the mapping $F$. We call $h$ a tangent vector to the set $M \subseteq X$ at $x_{*} \in M$ if there exist $\varepsilon>0$ and a function $r:[0, \varepsilon] \rightarrow X$ with the property that for $t \in[0, \varepsilon]$ we have $x_{*}+t h+r(t) \in M$ and $\|r(t)\|=o(t)$ as $t \rightarrow 0$ (for the sake of simplicity, the record $t \rightarrow 0$ will be omitted in the following text of paper). The set of all tangent vectors at $x_{*}$ is called the tangent cone to $M$ at $x_{*}$ and is denoted by $T_{1} M\left(x_{*}\right)$. A map $F: X \rightarrow Y$ is regular at $x_{*} \in X$ if $\operatorname{Im} F^{\prime}\left(x_{*}\right)=Y$. In the regular case, the tangent cone to the solution set coincides with the kernel of the first derivative of the map $F$. Recall the following theorem.

Theorem 1 (Classical Lyusternic theorem) Let $X$ and $Y$ be Banach spaces and a map $F: X \rightarrow Y$ be regular at $x_{*} \in X$. Then

$$
T_{1} M\left(x_{*}\right)=\operatorname{Ker} F^{\prime}\left(x_{*}\right) .
$$


The notion of regularity is generalized to the notion of the so-called $p$-regularity which will be described in the next section.

\section{Elements of $p$-regularity theory}

Assume that $x_{*} \in U \subseteq X, U$ is a neighborhood of an element $x_{*}$. Let a mapping $F: U \rightarrow Y$ be $p$-times Fréchet differentiable in $U$ and $\operatorname{Im} F^{\prime}\left(x_{*}\right) \neq Y$ (the regularity condition does not hold). In order to define the notion of $p$-regularity, let us define the so-called $p$-factor operator (see [1]). Assume that the space $Y$ is decomposed into a direct sum

$$
Y=Y_{1} \oplus \cdots \oplus Y_{p}
$$

where $Y_{1}=\operatorname{cl}\left(\operatorname{Im} F^{\prime}\left(x_{*}\right)\right)$ (the closure of the image of the first derivative of $F$ evaluated at $x_{*}$ ) and the next spaces are defined as follows. Let $Z_{2}$ be a closed complementary subspace to $Y_{1}$, that is, $Y=Y_{1} \oplus Z_{2}$ (we assume that such a closed complement exists), and let $P_{Z_{2}}$ : $Y \rightarrow Z_{2}$ be a projection operator onto $Z_{2}$ along $Y_{1}$. Let $Y_{2}=\operatorname{cl}\left(\operatorname{span} \operatorname{Im} P_{Z_{2}} F^{\prime \prime}\left(x_{*}\right)[\cdot]^{2}\right) \subseteq Z_{2}$ (the closure of the linear span of the image of the quadratic map $\left.P_{Z_{2}} F^{\prime \prime}\left(x_{*}\right)[\cdot]^{2}\right)$. More generally, define

$$
Y_{i}=\operatorname{cl}\left(\operatorname{span} \operatorname{Im} P_{Z_{i}} F^{(i)}\left(x_{*}\right)[\cdot]^{i}\right) \subseteq Z_{i}, \quad i=2, \ldots, p-1,
$$

where $Z_{i}$ is a closed complementary subspace to $Y_{1} \oplus \cdots \oplus Y_{i-1}, i=2, \ldots, p$, with respect to $Y$, and $P_{Z_{i}}: Y \rightarrow Z_{i}$ is a projection operator onto $Z_{i}$ along $Y_{1} \oplus \cdots \oplus Y_{i-1}, i=2, \ldots, p$. Finally, $Y_{p}=Z_{p}$. The order $p$ is chosen as the minimal number (if it exists) for which the above decomposition (5) holds.

Now, let us define the following mappings:

$$
f_{i}(x): U \rightarrow Y_{i}, \quad f_{i}(x)=P_{Y_{i}} F(x), \quad i=1, \ldots, p
$$

where $P_{Y_{i}}: Y \rightarrow Y_{i}$ is a projection operator from $Y$ along $Y_{1} \oplus \cdots \oplus Y_{i-1} \oplus Y_{i+1} \oplus \cdots \oplus Y_{p}$. Below we recall some important definitions for further considerations.

We introduce the following operator:

$$
\Psi_{p}\left(x_{*}, \cdot\right)=f_{1}^{\prime}\left(x_{*}\right)+f_{2}^{\prime \prime}\left(x_{*}\right)[\cdot]+\cdots+f_{p}^{(p)}\left(x_{*}\right)[\cdot]^{p-1} .
$$

This means that

$$
\Psi_{p}\left(x_{*}, h\right)=f_{1}^{\prime}\left(x_{*}\right)+f_{2}^{\prime \prime}\left(x_{*}\right)[h]+\cdots+f_{p}^{(p)}\left(x_{*}\right)[h]^{p-1} .
$$

Definition 2 Let $h \in X$. The linear operator $\Psi_{p}\left(x_{*}, h\right) \in \mathcal{L}(X, Y)$ is called the $p$-factor operator.

Sometimes it is convenient to use the following representation of the $p$-factor operator:

$$
\begin{aligned}
\Psi_{p}\left(x_{*}, h\right) & =\left(f_{1}^{\prime}\left(x_{*}\right), f_{2}^{\prime \prime}\left(x_{*}\right)[h], \ldots, f_{p}^{(p)}\left(x_{*}\right)[h]^{p-1}\right) \\
& =\left(P_{Y_{1}} F^{\prime}\left(x_{*}\right), P_{Y_{2}} F^{\prime \prime}\left(x_{*}\right)[h], \ldots, P_{Y_{p}} F^{(p)}\left(x_{*}\right)[h]^{p-1}\right)
\end{aligned}
$$

for $h \in X$. In this representation, we assume that $Y=Y_{1} \times \cdots \times Y_{p}$. 
We say that $F$ is completely degenerate at $x_{*}$ up to order $p$ if $F^{(i)}\left(x_{*}\right)=0, i=1, \ldots, p-1$. In the completely degenerate case, the $p$-factor operator is equal to $F^{(p)}\left(x_{*}\right)[h]^{p-1}$.

Definition 3 The $p$-kernel of the operator $\Psi_{p}\left(x_{*}, \cdot\right)$ is a set

$$
\begin{aligned}
H_{p}\left(x_{*}\right) & =\operatorname{Ker}^{p} \Psi_{p}\left(x_{*}, \cdot\right)=\left\{h \in X: \Psi_{p}\left(x_{*}, h\right)[h]=0\right\} \\
& =\left\{h \in X: f_{1}^{\prime}\left(x_{*}\right)[h]+f_{2}^{\prime \prime}\left(x_{*}\right)[h]^{2}+\cdots+f_{p}^{(p)}\left(x_{*}\right)[h]^{p}=0\right\} .
\end{aligned}
$$

Note that

$$
\operatorname{Ker}^{p} \Psi_{p}\left(x_{*}, \cdot\right)=\left\{\bigcap_{i=1}^{p} \operatorname{Ker}^{i} f_{i}^{(i)}\left(x_{*}\right)\right\} .
$$

In the completely degenerate case, $H_{p}\left(x^{*}\right)$ is equal to

$$
\operatorname{Ker}^{p} F^{(p)}\left(x_{*}\right)=\left\{h \in X: F^{(p)}\left(x_{*}\right)[h]^{p}=0\right\} .
$$

Definition 4 A mapping $F$ is called $p$-regular at $x_{*}$ along $h, p>1$, if $\operatorname{Im} \Psi_{p}\left(x_{*}, h\right)=Y$ (i.e., the operator $\Psi_{p}\left(x_{*}, h\right)$ is surjection).

Definition 5 A mapping $F$ is called $p$-regular at $x_{*}, p>1$, if it is $p$-regular along every $h$ belonging to the set $H_{p}\left(x_{*}\right) \backslash\{0\}$.

Note that if $H_{p}\left(x_{*}\right)=\{0\}$, then $F$ is automatically $p$-regular.

The following theorem gives a description of the tangent cone in the degenerate case.

Theorem 6 (Generalized Lyusternik theorem [1]) Let $X$ and $Y$ be Banach spaces, $F \in$ $\mathcal{C}^{p}(X, Y)$ be p-regular at $x_{*} \in U \subset X$. Then

$$
T_{1} M\left(x_{*}\right)=H_{p}\left(x_{*}\right)
$$

\section{Applications}

The following lemma will be important in the study of surjectivity of $p$-factor operators in the mentioned examples.

Lemma 7 Suppose that $Y=Y_{1} \oplus Y_{2}$, where $Y_{1}, Y_{2}$ are closed subspaces in $Y, A, B \in$ $\mathcal{L}(X, Y), \operatorname{Im} A=Y_{1}$. Let also $P_{2}$ be a projection onto $Y_{2}$ along $Y_{1}$. Then $\left(A+P_{2} B\right) X=Y \Leftrightarrow$ $\left(P_{2} B\right) \operatorname{Ker} A=Y_{2}$.

This lemma is a straightforward consequence of the following simple lemma.

Lemma 8 Suppose that $Y=Y_{1} \oplus Y_{2}$, where $Y_{1}, Y_{2}$ are closed subspaces in $Y, A_{1}, A_{2} \in$ $\mathcal{L}(X, Y), A_{1} X \subset Y_{1}, A_{2} X \subset Y_{2}$. Then $\left(A_{1}+A_{2}\right) X=Y$ iff $A_{1} \operatorname{Ker} A_{2}=Y_{1}$ and $A_{2} \operatorname{Ker} A_{1}=Y_{2}$.

The proof is obvious. Lemma 7 follows from Lemma 8 if we put $A_{1}=A$ and $A_{2}=P_{2} B$. 
Example 9 Now we apply the above theory to differential equation (2)

$$
F(x, \varepsilon)=x^{\prime \prime}+(1+\varepsilon)\left(x+x^{2}\right)=0,
$$

where $F: \mathcal{C}_{0}^{2}[0, \pi] \times \mathbb{R} \rightarrow \mathcal{C}[0, \pi]$ and $\mathcal{C}_{0}^{2}[0, \pi]=\left\{x \in \mathcal{C}^{2}[0, \pi]: x(0)=x(\pi)=0\right\}$. Observe that $x_{*}=(0,0)$ is a trivial solution of this equation.

The first derivative of the mapping $F$ is

$$
F^{\prime}(x, \varepsilon)=\left[F_{x}^{\prime}(x, \varepsilon), F_{\varepsilon}^{\prime}(x, \varepsilon)\right]=\left[\frac{d^{2}}{d t^{2}}+(1+\varepsilon)(1+2 x), x+x^{2}\right] .
$$

In our case, $F_{x}^{\prime}(0,0)=(\cdot)^{\prime \prime}+(\cdot)$ and $F_{\varepsilon}^{\prime}(0,0)=0$.

Note that $\operatorname{Ker} F_{x}^{\prime}(0,0)=\left\{x \in \mathcal{C}^{2}[0, \pi]: \frac{d^{2} x}{d t^{2}}+x=0\right\}$. The general solution of the equation $x^{\prime \prime}+x=0$ is $x(t)=c_{1} \cos t+c_{2} \sin t$. Taking into account the boundary condition, we obtain $c_{1}=0, x(t)=c_{2} \sin t$ and $\operatorname{Ker} F_{x}^{\prime}(0,0)=\operatorname{span}\{\sin t\}$.

The image of $F_{x}^{\prime}(0,0)$ is defined as follows:

$$
\begin{aligned}
\operatorname{Im} F_{x}^{\prime}(0,0) & =\left\{y \in \mathcal{C}[0, \pi]: \exists x \in \mathcal{C}^{2}[0, \pi] F_{x}^{\prime}(0,0) x=y, x(0)=x(\pi)=0\right\} \\
& =\left\{y \in \mathcal{C}[0, \pi]: \exists x \in \mathcal{C}^{2}[0, \pi] \text { such that } x^{\prime \prime}+x=y, x(0)=x(\pi)=0\right\} .
\end{aligned}
$$

The general solution of the above equation has the form

$$
x(t)=c_{1} \cos t+c_{2} \sin t-\cos t \int_{0}^{t} y(\tau) \sin \tau d \tau+\sin t \int_{0}^{t} y(\tau) \cos \tau d \tau .
$$

In view of the boundary conditions $x(0)=x(\pi)=0$, we obtain

$$
\operatorname{Im} F_{x}^{\prime}(0,0)=\left\{y \in \mathcal{C}[0, \pi]: \int_{0}^{\pi} y(\tau) \sin \tau d \tau=0\right\}
$$

One can easily show that the boundary value problem $x^{\prime \prime}+x=\sin t, x(0)=x(\pi)=0$ does not have a solution. Moreover, since $\int_{0}^{\pi} \sin ^{2}(t) d t \neq 0$, it follows that $y(t)=\sin t \notin$ $\operatorname{Im} F_{x}^{\prime}(0,0)$. This implies that the operator $F_{x}^{\prime}(0,0)$ is not surjective, i.e., $\operatorname{Im} F_{x}^{\prime}(0,0) \neq$ $\mathcal{C}[0, \pi]=Y$. Then $Y=Y_{1} \oplus Z_{2}$, where $Y_{1}=\operatorname{Im} F_{x}^{\prime}(0,0)$ and $Z_{2}=\operatorname{Ker} F_{x}^{\prime}(0,0)$.

The projector $P_{Z_{2}}: Y \rightarrow Z_{2}$ along $Y_{1}$ can be described as

$$
P_{Z_{2}} y=\frac{2}{\pi} \sin t \int_{0}^{\pi} y(\tau) \sin \tau d \tau, \quad y \in Y .
$$

Since

$$
\begin{aligned}
& F^{\prime \prime}(x, \varepsilon)=\left[\begin{array}{cc}
2(1+\varepsilon) & 1+2 x \\
1+2 x & 0
\end{array}\right], \quad F^{\prime \prime}(0,0)=\left[\begin{array}{ll}
2 & 1 \\
1 & 0
\end{array}\right], \\
& F^{\prime \prime}(0,0)\left[h_{z}, h_{\varepsilon}\right]=\left[2 h_{z}+h_{\varepsilon}, h_{z}\right], \quad F^{\prime \prime}(0,0)\left[h_{z}, h_{\varepsilon}\right]^{2}=2 h_{z}^{2}+2 h_{z} h_{\varepsilon},
\end{aligned}
$$

then

$$
\begin{aligned}
Y_{2} & =\operatorname{span}\left(\operatorname{Im} P_{Z_{2}} F^{\prime \prime}(0,0)[\cdot]^{2}\right) \\
& =\operatorname{span}\left\{y(t) \in Y: y(t)=\frac{2}{\pi} \sin t \int_{0}^{\pi} F^{\prime \prime}(0,0)[\cdot]^{2} \sin \tau d \tau\right\}=\operatorname{span}\{\sin t\}=Z_{2}
\end{aligned}
$$


and

$$
Y=\mathcal{C}[0, \pi]=Y_{1} \oplus Y_{2}=\operatorname{Im} F_{x}^{\prime}(0,0) \oplus \operatorname{Ker} F_{x}^{\prime}(0,0)
$$

Consequently, $\left[\operatorname{Im} F_{x}^{\prime}(0,0)\right]^{\perp}=\operatorname{Ker} F_{x}^{\prime}(0,0)$.

Using $p$-regularity theory and the generalized Lyusternik theorem, we obtain the following assertion. If the mapping $F$ is $p$-regular $(p=2)$ at the point $x_{*}=(0,0)$ with respect to the element $h=\left[h_{z}, h_{\varepsilon}\right]$, where $h_{z}=z \sin t, z \in \mathbb{R}, h_{\varepsilon}=\bar{\varepsilon}$, then there exist solutions $x=x(t, \varepsilon)=\varepsilon z \sin t+r(\varepsilon),\|r(\varepsilon)\|=o(\varepsilon)$ of equation (2) for $\varepsilon \in(-\sigma, \sigma)$, where $\sigma>0$ is sufficiently small. Below we will describe a 2-factor operator and show its surjectivity.

Let $P_{1}=P_{Y_{1}}$ be a projection onto $Y_{1}=\operatorname{Im} F_{x}^{\prime}(0,0)$ and $P_{2}=P_{Y_{2}}$ be a projection onto $Y_{2}=$ $\operatorname{Ker} F_{x}^{\prime}(0,0)$ along $Y_{1}$. The 2-factor operator has the form

$$
\begin{aligned}
\Psi_{2}\left((0,0),\left[h_{z}, h_{\varepsilon}\right]\right) & =f_{1}^{\prime}(0,0)+f_{2}^{\prime \prime}(0,0)\left[h_{z}, h_{\varepsilon}\right] \\
& =P_{1} F_{x}^{\prime}(0,0)+P_{2} F^{\prime \prime}(0,0)\left[h_{z}, h_{\varepsilon}\right] \\
& =F_{x}^{\prime}(0,0)+P_{2} F^{\prime \prime}(0,0)\left[h_{z}, h_{\varepsilon}\right]
\end{aligned}
$$

because $P_{1} F_{x}^{\prime}=F_{x}^{\prime}$.

Using the second derivative of $F$, we obtain, for $\left[h_{u}, h_{\lambda}\right] \in \mathcal{C}^{2}[0, \pi] \times \mathbb{R}$,

$$
\begin{aligned}
\Psi_{2}\left((0,0),\left[h_{z}, h_{\varepsilon}\right]\right)\left[h_{u}, h_{\lambda}\right] & =\frac{d^{2} h_{u}}{d t^{2}}+h_{u}+P_{2}\left[2 h_{z} h_{u}+h_{\varepsilon} h_{u}+h_{z} h_{\lambda}\right] \\
& =\frac{d^{2} h_{u}}{d t^{2}}+h_{u}+\frac{2}{\pi}\left\langle 2 h_{z} h_{u}+h_{\varepsilon} h_{u}+h_{z} h_{\lambda}, \sin t\right\rangle \sin t \\
& =\frac{d^{2} h_{u}}{d t^{2}}+h_{u}+\frac{2}{\pi} \sin t \int_{0}^{\pi}\left(2 h_{z} h_{u}+h_{\varepsilon} h_{u}+h_{z} h_{\lambda}\right) \sin t d t
\end{aligned}
$$

Now we describe the 2-kernel of the operator $\Psi_{2}((0,0), \cdot)$

$$
H_{2}(0,0)=\left\{h=\left[h_{z}, h_{\varepsilon}\right] \in \mathcal{C}^{2}[0, \pi] \times \mathbb{R}: \Psi_{2}((0,0), h)[h]=0\right\} .
$$

Assume that $h=\left[h_{z}, h_{\varepsilon}\right] \in \operatorname{Ker} F_{x}^{\prime}(0,0)$, i.e., $F_{x}^{\prime}(0,0)\left[h_{z}, h_{\varepsilon}\right]=0$. Then $h_{z}=z \sin t, z \in \mathbb{R}, h_{\varepsilon} \in$ $\mathbb{R}$ and

$$
\begin{aligned}
& \Psi_{2}\left((0,0),\left[h_{z}, h_{\varepsilon}\right]\right)\left[h_{z}, h_{\varepsilon}\right]=P_{2} F^{\prime \prime}(0,0)\left[h_{z}, h_{\varepsilon}\right]^{2}=\frac{2}{\pi} \sin t \int_{0}^{\pi}\left(2 h_{z}^{2}+2 h_{z} h_{\varepsilon}\right) \sin t d t=0 \\
& \Leftrightarrow \quad \int_{0}^{\pi}\left(2 h_{z}^{2}+2 h_{z} h_{\varepsilon}\right) \sin t d t=0 \\
& \quad \Leftrightarrow \quad z^{2} \int_{0}^{\pi} \sin ^{3} t d t+z h_{\varepsilon} \int_{0}^{\pi} \sin ^{2} t d t=0 \\
& \Leftrightarrow \quad \frac{4}{3} z^{2}+\frac{\pi}{2} z h_{\varepsilon}=0 \quad \Leftrightarrow \quad z=0 \vee z=-\frac{3}{8} \pi h_{\varepsilon} \\
& \Leftrightarrow \quad h_{z}=0 \vee h_{z}=-\frac{3}{8} \pi h_{\varepsilon} \sin t .
\end{aligned}
$$

This implies that $H_{2}(0,0)=\{[0, \bar{\varepsilon}]\} \cup\left\{\left[-\frac{3}{8} \pi \bar{\varepsilon} \sin t, \bar{\varepsilon}\right]\right\}$, where $h_{\varepsilon}=\bar{\varepsilon}$. 
Next we verify that the mapping $\Psi_{2}\left((0,0),\left[h_{z}, h_{\varepsilon}\right]\right)$ is surjective onto $\mathcal{C}[0, \pi]$ for elements belonging to the set $H_{2}(0,0)$, that is,

$$
\forall y \in \mathcal{C}[0, \pi] \exists\left[h_{u}, h_{\lambda}\right] \in \mathcal{C}^{2}[0, \pi] \times \mathbb{R} \text { such that } \Psi_{2}\left((0,0),\left[h_{z}, h_{\varepsilon}\right]\right)\left[h_{u}, h_{\lambda}\right]=y .
$$

Consider the first case $\left[h_{z}, h_{\varepsilon}\right]=[0, \bar{\varepsilon}], \bar{\varepsilon} \neq 0$.

Assume that

$$
\frac{d^{2} h_{u}(t)}{d t^{2}}+h_{u}(t)+\frac{2}{\pi} \sin t \int_{0}^{\pi} \bar{\varepsilon} h_{u}(t) \sin t d t=y=y_{1}+y_{2}
$$

where

$$
\begin{aligned}
& y_{1}=\frac{d^{2} h_{u}(t)}{d t^{2}}+h_{u}(t)=P_{1} y \in Y_{1}=\operatorname{Im} F_{x}^{\prime}(0,0), \\
& y_{2}=\frac{2}{\pi} \sin t \int_{0}^{\pi} \bar{\varepsilon} h_{u}(t) \sin t d t=P_{2} y \in Y_{2}=\operatorname{Ker} F_{x}^{\prime}(0,0) .
\end{aligned}
$$

Putting $y_{2}=a \sin t$ and using Lemma 7, it suffices to take $h_{u}=b \sin t \in \operatorname{Ker} F_{x}^{\prime}(0,0)$. Then from (6) we obtain $y_{1}=0$ and $\frac{2}{\pi} \bar{\varepsilon} \sin t \int_{0}^{\pi} b \sin ^{2} t d t=a \sin t$ from where $b=\frac{a}{\bar{\varepsilon}}, h_{u}=\frac{a}{\bar{\varepsilon}} \sin t$. The solutions of equation (6) exist, and hence $\Psi_{2}\left((0,0),\left[h_{z}, h_{\varepsilon}\right]\right)$ is surjective.

Verify the second case $\left[h_{z}, h_{\varepsilon}\right]=\left[-\frac{3}{8} \pi \bar{\varepsilon} \sin t, \bar{\varepsilon}\right], \bar{\varepsilon} \neq 0$. Consider the equation

$$
\begin{aligned}
& \frac{d^{2} h_{u}(t)}{d t^{2}}+h_{u}(t)+\frac{2}{\pi} \sin t \int_{0}^{\pi}\left[2 \bar{\varepsilon}\left(-\frac{3}{8} \pi \sin t\right) h_{u}(t)+\bar{\varepsilon} h_{u}(t)\right. \\
& \left.+\bar{\varepsilon}\left(-\frac{3}{8} \pi \sin t\right) h_{\lambda}\right] \sin t d t=y=y_{1}+y_{2} .
\end{aligned}
$$

Putting $y_{2}=a \sin t$, using Lemma 7 and taking $h_{u}=b \sin t \in \operatorname{Ker} F_{x}^{\prime}(0,0)$, we have $y_{1}=0$ and

$$
-\frac{3 \pi}{8} h_{\lambda} \bar{\varepsilon} \sin t+\frac{2}{\pi} \bar{\varepsilon} \sin t \int_{0}^{\pi}\left(-\frac{3}{4} \pi \sin ^{2} t+\sin t\right) b \sin t d t=a \sin t .
$$

Hence $b=-\frac{3}{8} \pi h_{\lambda}-\frac{a}{\bar{\varepsilon}}$, and $h_{u}=\left(-\frac{3}{8} \pi h_{\lambda}-\frac{a}{\bar{\varepsilon}}\right) \sin t$. The solutions of equation (7) exist, hence $\Psi_{2}\left((0,0),\left[h_{z}, h_{\varepsilon}\right]\right)$ is surjective in this case too. Therefore the mapping $F$ is 2-regular at the point $x_{*}=(0,0)$ with respect to the element $h=\left[h_{z}, h_{\varepsilon}\right]$.

Using the generalized Lyusternik theorem, we can describe the solutions belonging to the tangent cone $T_{1} M(0,0)$. For $\left[h_{z}, h_{\varepsilon}\right]=[0, \bar{\varepsilon}]$ we have $x_{1}(t, \varepsilon)=r_{1}(\varepsilon)$ and for $\left[h_{z}, h_{\varepsilon}\right]=$ $\left[-\frac{3}{8} \pi \bar{\varepsilon} \sin t, \bar{\varepsilon}\right]$ we have $x_{2}(t, \varepsilon)=-\frac{3}{8} \pi \varepsilon \sin t+r_{2}(\varepsilon)$, where $\left\|r_{i}(\varepsilon)\right\|=o(\varepsilon)$ for $i=1,2$ and $\varepsilon \in$ $(-\sigma, \sigma), \sigma>0$ is sufficiently small. Thus we obtain the following theorem.

Theorem 10 Equation (2) has two solutions $x_{i}(t, \varepsilon), i=1,2$, such that

$$
\begin{aligned}
& x_{1}(t, \varepsilon)=r_{1}(\varepsilon), \\
& x_{2}(t, \varepsilon)=-\frac{3}{8} \pi \varepsilon \sin t+r_{2}(\varepsilon),
\end{aligned}
$$

where $\left\|r_{i}(\varepsilon)\right\|=o(\varepsilon)$ for $i=1,2$ and $\varepsilon \in(-\sigma, \sigma), \sigma>0$ is sufficiently small. 
Example 11 Equation (3), i.e.,

$$
F(x, \varepsilon)=x^{\prime \prime}+x+\varepsilon x^{p-1}+x^{p}=0,
$$

where $F: \mathcal{C}_{0}^{2}[0, \pi] \times \mathbb{R} \rightarrow \mathcal{C}[0, \pi], p \geq 2$, can be investigated analogously. Similarly, we obtain the following result.

Theorem 12 Equation (3) has a nonzero solution $x(t, \varepsilon)$ such that

$$
x(t, \varepsilon)=-\frac{\varepsilon}{p} c_{p} \sin t+r(\varepsilon),
$$

where

$$
c_{p}=\frac{\int_{0}^{\pi} \sin ^{p} t d t}{\int_{0}^{\pi} \sin ^{p+1} t d t}, \quad\|r(\varepsilon)\|=o(\varepsilon)
$$

and $\varepsilon \in(-\sigma, \sigma), \sigma>0$ is sufficiently small.

Example 13 Consider now equation (4)

$$
F(u, \varepsilon)=\Delta u-(\varepsilon-10) g(u)=0,
$$

where $F: \mathcal{C}_{0}^{2}[\Omega] \times \mathbb{R} \rightarrow \mathcal{C}[\Omega], \Omega=[0, \pi] \times[0, \pi], \Delta$ is the Laplacian, with the assumption $g(0)=0, g^{\prime}(0)=1, g^{\prime \prime}(0) \neq 0$. The point $x_{*}=(0,0)$ is the trivial solution of the above equation.

We put $u=u(y)=u\left(y_{1}, y_{2}\right)$.

The first derivative of the mapping $F$ is the following:

$$
F^{\prime}(u, \varepsilon)=\left[F_{u}^{\prime}(u, \varepsilon), F_{\varepsilon}^{\prime}(u, \varepsilon)\right]=\left[\Delta-(\varepsilon-10) g^{\prime}(u),-g(u)\right] .
$$

In our case $F_{u}^{\prime}(0,0)=\Delta+10$.

Note that $\operatorname{Ker} F_{u}^{\prime}(0,0)=\left\{u \in \mathcal{C}_{0}^{2}[\Omega]: \Delta u+10 u=0\right\}$. This space is spanned by the $L^{2}$ orthonormal functions (see [8], p.424)

$$
u_{1}=\frac{2}{\pi} \sin 3 y_{1} \sin y_{2}, \quad u_{2}=\frac{2}{\pi} \sin y_{1} \sin 3 y_{2}
$$

(that is, $\left\langle u_{i}, u_{j}\right\rangle=\int_{\Omega} u_{i} u_{j} d y_{1} d y_{2}$ and $\int_{\Omega} u_{i}^{2} d y_{1} d y_{2}=1, \int_{\Omega} u_{i} u_{j} d y_{1} d y_{2}=0$ for $\left.i \neq j\right)$. Moreover,

$$
\mathcal{C}[\Omega]=\operatorname{Im} F_{u}^{\prime}(0,0) \oplus \operatorname{Ker} F_{u}^{\prime}(0,0)
$$

and

$$
\left[\operatorname{Im} F_{u}^{\prime}(0,0)\right]^{\perp}=\operatorname{Ker} F_{u}^{\prime}(0,0)
$$

From $p$-regularity theory and the generalized Lyusternik theorem, we obtain the following assertion. If the mapping $F$ is $p$-regular at the point $x_{*}=(0,0)$ with respect to the element $h=\left[h_{z}, h_{\varepsilon}\right]$, where $z=\left(z_{1}, z_{2}\right), h_{z}=z_{1} u_{1}+z_{2} u_{2}, h_{\varepsilon}=\bar{\varepsilon}$, then there exist solutions $u=u(y, \varepsilon)=$ 
$\varepsilon\left(z_{1} u_{1}+z_{2} u_{2}\right)+r(\varepsilon),\|r(\varepsilon)\|=o(\varepsilon)$ of equation (4) for $\varepsilon \in(-\sigma, \sigma)$, where $\sigma>0$ is sufficiently small.

Let $P_{1}=P_{Y_{1}}$ be the projection of $Y$ onto $Y_{1}=\operatorname{Im} F_{u}^{\prime}(0,0)$, and let $P_{2}=P_{Y_{2}}$ be the projection of $Y$ onto $Y_{2}=\operatorname{Ker} F_{u}^{\prime}(0,0)$ along $Y_{1}$. We define the 2-factor operator:

$$
\begin{aligned}
\Psi_{2}((0,0), h) & =\Psi_{2}\left((0,0),\left[h_{z}, h_{\varepsilon}\right]\right)=f_{1}^{\prime}(0,0)+f_{2}^{\prime \prime}(0,0)\left[h_{z}, h_{\varepsilon}\right] \\
& =P_{1} F_{u}^{\prime}(0,0)+P_{2} F^{\prime \prime}(0,0)\left[h_{z}, h_{\varepsilon}\right] \\
& \left.=F_{u}^{\prime}(0,0)+P_{2} F^{\prime \prime}(0,0)\left[h_{z}, h_{\varepsilon}\right] \quad \text { (because } P_{1} F_{u}^{\prime}=F_{u}^{\prime}\right) .
\end{aligned}
$$

Using the second derivative of $F$, that is,

$$
\begin{aligned}
& F^{\prime \prime}(u, \varepsilon)=\left[\begin{array}{cc}
-(\varepsilon-10) g^{\prime \prime}(u) & -g^{\prime}(u) \\
-g^{\prime}(u) & 0
\end{array}\right], \quad F^{\prime \prime}(0,0)=\left[\begin{array}{cc}
10 g^{\prime \prime}(0) & -1 \\
-1 & 0
\end{array}\right], \\
& F^{\prime \prime}(0,0)\left[h_{z}, h_{\varepsilon}\right]=\left[\begin{array}{cc}
10 g^{\prime}(0) & -1 \\
-1 & 0
\end{array}\right]\left[h_{z}, h_{\varepsilon}\right]=\left[10 g^{\prime \prime}(0) h_{z}-h_{\varepsilon},-h_{z}\right],
\end{aligned}
$$

we obtain, for $\left[h_{u}, h_{\lambda}\right] \in \mathcal{C}_{0}^{2}[\Omega] \times \mathbb{R}$,

$$
F^{\prime \prime}(0,0)\left[h_{z}, h_{\varepsilon}\right]\left[h_{u}, h_{\lambda}\right]=\left[10 g^{\prime \prime}(0) h_{z}-h_{\varepsilon},-h_{z}\right]\left[h_{u}, h_{\lambda}\right]=10 g^{\prime \prime}(0) h_{z} h_{u}-h_{\varepsilon} h_{u}-h_{z} h_{\lambda},
$$

and hence

$$
F^{\prime \prime}(0,0)\left[h_{z}, h_{\varepsilon}\right]^{2}=10 g^{\prime \prime}(0) h_{z}^{2}-2 h_{z} h_{\varepsilon}
$$

From the above calculations it follows that the value of the 2-factor operator $\Psi_{2}((0,0)$, $\left.\left[h_{z}, h_{\varepsilon}\right]\right)$ on an element $x=\left[h_{u}, h_{\lambda}\right] \in \mathcal{C}_{0}^{2}[\Omega] \times \mathbb{R}$ is equal to

$$
\begin{aligned}
\Psi_{2}((0,0), h)[x] & =\Psi_{2}\left((0,0),\left[h_{z}, h_{\varepsilon}\right]\right)\left[h_{u}, h_{\lambda}\right] \\
& =\Delta h_{u}+10 h_{u}+P_{2}\left[10 g^{\prime \prime}(0) h_{z} h_{u}-h_{\varepsilon} h_{u}-h_{z} h_{\lambda}\right] \\
& =\Delta h_{u}+10 h_{u}+\sum_{i=1}^{2}\left\langle 10 g^{\prime \prime}(0) h_{z} h_{u}-h_{\varepsilon} h_{u}-h_{z} h_{\lambda}, u_{i}\right) u_{i} .
\end{aligned}
$$

Now we describe the 2-kernel of the operator $\Psi_{2}((0,0), \cdot)$, which is the set

$$
H_{2}(0,0)=\left\{h=\left[h_{z}, h_{\varepsilon}\right] \in \mathcal{C}_{0}^{2}[\Omega] \times \mathbb{R}: \Psi_{2}((0,0), h)[h]=0\right\} .
$$

Assuming that $\left[h_{z}, h_{\varepsilon}\right]=\left[z_{1} u_{1}+z_{2} u_{2}, \bar{\varepsilon}\right] \in \operatorname{Ker} F_{u}^{\prime}(0,0)$, we have

$$
F_{u}^{\prime}(0,0)\left[h_{z}, h_{\varepsilon}\right]=0
$$

and

$$
\begin{aligned}
& \Psi_{2}\left((0,0),\left[h_{z}, h_{\varepsilon}\right]\right)\left[h_{z}, h_{\varepsilon}\right] \\
& \quad=P_{2} F^{\prime \prime}(0,0)\left[h_{z}, h_{\varepsilon}\right]^{2}=\sum_{i=1}^{2}\left\langle F^{\prime \prime}(0,0)\left[h_{z}, h_{\varepsilon}\right]^{2}, u_{i}\right\rangle u_{i}
\end{aligned}
$$




$$
\begin{aligned}
& =\sum_{i=1}^{2} u_{i} \int_{\Omega}\left(10 g^{\prime \prime}(0) h_{z}^{2}-2 h_{z} h_{\varepsilon}\right) u_{i} d y_{1} d y_{2} \\
& =\sum_{i=1}^{2} u_{i} \int_{\Omega}\left[10 g^{\prime \prime}(0)\left(z_{1} u_{1}+z_{2} u_{2}\right)^{2}-2\left(z_{1} u_{1}+z_{2} u_{2}\right) \bar{\varepsilon}\right] u_{i} d y_{1} d y_{2} \\
& =\sum_{i=1}^{2} u_{i}\left[10 g^{\prime \prime}(0) \int_{\Omega} u_{i}\left(z_{1} u_{1}+z_{2} u_{2}\right)^{2} d y_{1} d y_{2}-2 \bar{\varepsilon} \int_{\Omega} u_{i}\left(z_{1} u_{1}+z_{2} u_{2}\right) d y_{1} d y_{2}\right] \\
& =\sum_{i=1}^{2} u_{i}\left[10 g^{\prime \prime}(0) \int_{\Omega} u_{i}\left(z_{1} u_{1}+z_{2} u_{2}\right)^{2} d y_{1} d y_{2}-2 \bar{\varepsilon} z_{i}\right]
\end{aligned}
$$

Next, introducing the quadratic forms

$$
Q_{i}\left(h_{z}, \bar{\varepsilon}\right)=Q_{i}\left(z_{1}, z_{2}, \bar{\varepsilon}\right)=10 g^{\prime \prime}(0) \int_{\Omega} u_{i}\left(z_{1} u_{1}+z_{2} u_{2}\right)^{2} d y_{1} d y_{2}-2 \bar{\varepsilon} z_{i}, \quad i=1,2,
$$

we obtain the following system of equations for elements belonging to the set $H_{2}(0,0)$ :

$$
\left\{\begin{array}{l}
Q_{1}\left(h_{z}, \bar{\varepsilon}\right)=Q_{1}\left(z_{1}, z_{2}, \bar{\varepsilon}\right)=10 g^{\prime \prime}(0) \int_{\Omega} u_{1}\left(z_{1} u_{1}+z_{2} u_{2}\right)^{2} d y_{1} d y_{2}-2 \bar{\varepsilon} z_{1}=0 \\
Q_{2}\left(h_{z}, \bar{\varepsilon}\right)=Q_{2}\left(z_{1}, z_{2}, \bar{\varepsilon}\right)=10 g^{\prime \prime}(0) \int_{\Omega} u_{2}\left(z_{1} u_{1}+z_{2} u_{2}\right)^{2} d y_{1} d y_{2}-2 \bar{\varepsilon} z_{2}=0
\end{array}\right.
$$

or, equivalently,

$$
\left\{\begin{array}{l}
10 g^{\prime \prime}(0) \int_{\Omega}\left(u_{1}^{3} z_{1}^{2}+2 z_{1} z_{2} u_{1}^{2} u_{2}+z_{2}^{2} u_{1} u_{2}^{2}\right) d y_{1} d y_{2}-2 \bar{\varepsilon} z_{1}=0 \\
10 g^{\prime \prime}(0) \int_{\Omega}\left(u_{1} u_{2}^{2} z_{1}^{2}+2 z_{1} z_{2} u_{1} u_{2}^{2}+z_{2}^{2} u_{2}^{3}\right) d y_{1} d y_{2}-2 \bar{\varepsilon} z_{2}=0
\end{array}\right.
$$

Simple calculations give us

$$
\begin{aligned}
& a=\int_{\Omega} u_{1}^{3} d y_{1} d y_{2}=\int_{\Omega} u_{2}^{3} d y_{1} d y_{2}=\frac{16}{27} \cdot \frac{8}{\pi^{3}}, \\
& b=\int_{\Omega} u_{1}^{2} u_{2} d y_{1} d y_{2}=\int_{\Omega} u_{1} u_{2}^{2} d y_{1} d y_{2}=-\frac{48}{175} \cdot \frac{8}{\pi^{3}},
\end{aligned}
$$

and we obtain the following form of system (8):

$$
\left\{\begin{array}{l}
10 g^{\prime \prime}(0)\left(a z_{1}^{2}+2 b z_{1} z_{2}+b z_{2}^{2}\right)-2 \bar{\varepsilon} z_{1}=0 \\
10 g^{\prime \prime}(0)\left(b z_{1}^{2}+2 b z_{1} z_{2}+a z_{2}^{2}\right)-2 \bar{\varepsilon} z_{2}=0
\end{array}\right.
$$

or putting $C_{i}^{j k}=10 g^{\prime \prime}(0) \int_{\Omega} u_{i} u_{j} u_{k} d y_{1} d y_{2}$, we get

$$
\sum_{j, k=1}^{2} C_{i}^{j k} z_{j} z_{k}-2 \bar{\varepsilon} z_{i}=0, \quad i=1,2
$$

Note that if $M\left(z_{1}, z_{2}\right)$ is the $2 \times 2$ symmetric matrix whose $(i, j)$ entry is

$$
a_{i j}=\sum_{m=1}^{2}\left(\int_{\Omega} u_{i} u_{j} u_{m} d y_{1} d y_{2}\right) z_{m}
$$


that is,

$$
M\left(z_{1}, z_{2}\right)=\left[\begin{array}{ll}
a z_{1}+b z_{2} & b z_{1}+b z_{2} \\
b z_{1}+b z_{2} & b z_{1}+a z_{2}
\end{array}\right],
$$

then system (9) is equivalent to the following equation:

$$
\left[10 g^{\prime \prime}(0) M(z)-2 \bar{\varepsilon} I\right]\left[\begin{array}{l}
z_{1} \\
z_{2}
\end{array}\right]=0, \quad \text { where } I=\left[\begin{array}{ll}
1 & 0 \\
0 & 1
\end{array}\right] .
$$

Thus we get the following condition: elements $\left[\bar{z}_{1} u_{1}+\bar{z}_{2} u_{2}, \bar{\varepsilon}\right] \in H_{2}(0,0)$ (that is, elements $\left(\bar{z}_{1}, \bar{z}_{2}, \bar{\varepsilon}\right)$ are the solutions of system (9)) if and only if either $\left(\bar{z}_{1}, \bar{z}_{2}\right)$ is an eigenvector of $10 g^{\prime \prime}(0) M(z)$ corresponding to the eigenvalue $2 \bar{\varepsilon}$ or $\left(\bar{z}_{1}, \bar{z}_{2}\right)=(0,0)$. We will prove that for $\left[0 u_{1}+0 u_{2}, \bar{\varepsilon}\right] \in H_{2}(0,0)$, the mapping $F$ is always 2-regular and for any other $\left[\bar{z}_{1} u_{1}+\bar{z}_{2} u_{2}, \bar{\varepsilon}\right] \in H_{2}(0,0), F$ is 2-regular if $\bar{\varepsilon}$ is not an eigenvalue of $10 g^{\prime \prime}(0) M(z)$.

Note that $Q\left(z_{1}, z_{2}, \bar{\varepsilon}\right)=\left(Q_{1}\left(z_{1}, z_{2}, \bar{\varepsilon}\right), Q_{2}\left(z_{1}, z_{2}, \bar{\varepsilon}\right)\right)$ is a quadratic mapping associated to the 2 -factor operator $\Psi_{2}((0,0), h)$. If $\left[\bar{z}_{1} u_{1}+\bar{z}_{2} u_{2}, \bar{\varepsilon}\right] \in H_{2}(0,0)$, then the first differentiation of $Q$ gives us the matrix form of the operator $\Psi_{2}((0,0), h)$. Consider the calculations:

$$
\begin{aligned}
& Q^{\prime}\left(z_{1}, z_{2}, \bar{\varepsilon}\right)=\left[\begin{array}{ccc}
10 g^{\prime \prime}(0)\left(2 z_{1} a+2 z_{2} b\right)-2 \bar{\varepsilon} & 10 g^{\prime \prime}(0)\left(2 z_{1} b+2 z_{2} b\right) & -2 z_{1} \\
10 g^{\prime \prime}(0)\left(2 z_{1} b+2 z_{2} b\right) & 10 g^{\prime \prime}(0)\left(2 z_{1} b+2 z_{2} a\right)-2 \bar{\varepsilon} & -2 z_{2}
\end{array}\right], \\
& Q^{\prime \prime}\left(z_{1}, z_{2}, \bar{\varepsilon}\right)=\left[\begin{array}{c}
{\left[\begin{array}{ccc}
10 g^{\prime \prime}(0) 2 a & 10 g^{\prime \prime}(0) 2 b & -2 \\
10 g^{\prime \prime}(0) 2 b & 10 g^{\prime \prime}(0) 2 b & 0 \\
-2 & 0 & 0
\end{array}\right]} \\
{\left[\begin{array}{ccc}
10 g^{\prime \prime}(0) 2 b & 10 g^{\prime \prime}(0) 2 b & 0 \\
10 g^{\prime \prime}(0) 2 b & 10 g^{\prime \prime}(0) 2 a & -2 \\
0 & -2 & 0
\end{array}\right]}
\end{array}\right],
\end{aligned}
$$

and $\Psi_{2}((0,0), h)=Q^{\prime \prime}\left(z_{1}, z_{2}, \bar{\varepsilon}\right)\left[\begin{array}{c}z_{1} \\ z_{2} \\ \bar{\varepsilon}\end{array}\right]$.

It follows that

$$
\Psi_{2}((0,0), h)=\left[\begin{array}{ccc}
10 g^{\prime \prime}(0)\left(2 z_{1} a+2 z_{2} b\right)-2 \bar{\varepsilon} & 10 g^{\prime \prime}(0)\left(2 z_{1} b+2 z_{2} b\right) & -2 z_{1} \\
10 g^{\prime \prime}(0)\left(2 z_{1} b+2 z_{2} b\right) & 10 g^{\prime \prime}(0)\left(2 z_{1} b+2 z_{2} a\right)-2 \bar{\varepsilon} & -2 z_{2}
\end{array}\right],
$$

that is, $\Psi_{2}((0,0), h)=Q^{\prime}$.

For an arbitrary element $h=\left(z_{1}, z_{2}, \bar{\varepsilon}\right) \in H_{2}(0,0)$, we examine the surjectivity of the mapping $\Psi_{2}((0,0), h)$, that is, the following condition:

$\forall \eta \in \mathcal{C}[\Omega] \exists \xi \in \mathcal{C}_{0}^{2}[\Omega] \times \mathbb{R}$ such that $\Psi_{2}((0,0), h)[\xi]=\eta$

For $\xi=\left(\xi_{1}, \xi_{2}, \lambda\right), \eta=\left(\eta_{1}, \eta_{2}\right)$, we have

$$
\begin{aligned}
& \Psi_{2}((0,0), h)\left[\begin{array}{l}
\xi_{1} \\
\xi_{2} \\
\lambda
\end{array}\right] \\
& \quad=\left[\begin{array}{c}
{\left[10 g^{\prime \prime}(0)\left(2 \bar{z}_{1} a+2 \bar{z}_{2} b\right)-2 \bar{\varepsilon}\right] \xi_{1}+\left[10 g^{\prime \prime}(0)\left(2 \bar{z}_{1} b+2 \bar{z}_{2} b\right)\right] \xi_{2}-2 \bar{z}_{1} \lambda} \\
10 g^{\prime \prime}(0)\left(2 \bar{z}_{1} b+2 \bar{z}_{2} b\right) \xi_{1}+\left[10 g^{\prime \prime}(0)\left(2 \bar{z}_{1} b+2 \bar{z}_{2} a\right)-2 \bar{\varepsilon}\right] \xi_{2}-2 \bar{z}_{2} \lambda
\end{array}\right]=\left[\begin{array}{l}
\eta_{1} \\
\eta_{2}
\end{array}\right],
\end{aligned}
$$


or

$$
\left[10 g^{\prime \prime}(0)\left[\begin{array}{ll}
2 a \bar{z}_{1}+2 b \bar{z}_{2} & 2 b \bar{z}_{1}+2 b \bar{z}_{2} \\
2 b \bar{z}_{1}+2 b \bar{z}_{2} & 2 b \bar{z}_{1}+2 a \bar{z}_{2}
\end{array}\right]-2 \bar{\varepsilon}\left[\begin{array}{ll}
1 & 0 \\
0 & 1
\end{array}\right]\right]\left[\begin{array}{l}
\xi_{1} \\
\xi_{2}
\end{array}\right]-2 \lambda\left[\begin{array}{l}
\bar{z}_{1} \\
\bar{z}_{2}
\end{array}\right]=\left[\begin{array}{l}
\eta_{1} \\
\eta_{2}
\end{array}\right]
$$

and

$$
2\left[10 g^{\prime \prime}(0) M(z)-\bar{\varepsilon} I\right]\left[\begin{array}{l}
\xi_{1} \\
\xi_{2}
\end{array}\right]=2 \lambda\left[\begin{array}{l}
\bar{z}_{1} \\
\bar{z}_{2}
\end{array}\right]+\left[\begin{array}{l}
\eta_{1} \\
\eta_{2}
\end{array}\right]
$$

Observe that for $\left[0 u_{1}+0 u_{2}, \bar{\varepsilon}\right], \bar{\varepsilon} \neq 0, F$ is always 2-regular (since the matrix (10) has the maximal rank). Now put $\bar{\varepsilon}=0$ in (9). Then we get $\bar{z}_{1}=\bar{z}_{2}=0$. This means that in this case $H_{2}(0,0)=\{0\}$ and then $F$ is 2-regular (in this trivial case, the equation $F(u, \varepsilon)=0$ has the unique solution $u=0)$. Consider the other $\left[\bar{z}_{1} u_{1}+\bar{z}_{2} u_{2}, \bar{\varepsilon}\right] \in H_{2}(0,0)$. Then equation (11) has the solution if $\operatorname{det}\left[10 g^{\prime \prime}(0) M(z)-\bar{\varepsilon} I\right] \neq 0$. This means that $\bar{\varepsilon}$ is not an eigenvalue of $10 g^{\prime \prime}(0) M(z)$.

From the above consideration, we obtain the following sufficient condition for 2-regularity: the matrix $10 g^{\prime \prime}(0) M(z)$ has no eigenvalues $\bar{\varepsilon}$ and $2 \bar{\varepsilon}$. This condition can be tested using the notion of resultant. We evidently have $10 g^{\prime \prime}(0) \neq 0$. Let $\lambda$ and $2 \lambda$ be the eigenvalues, and let $\lambda \neq 0$. Then we obtain

$$
\begin{aligned}
\operatorname{det}\left[M\left(z_{1}, z_{2}\right)-\lambda I\right] & =\operatorname{det}\left[\left[\begin{array}{ll}
a z_{1}+b z_{2} & b z_{1}+b z_{2} \\
b z_{1}+b z_{2} & b z_{1}+a z_{2}
\end{array}\right]-\lambda\left[\begin{array}{ll}
1 & 0 \\
0 & 1
\end{array}\right]\right] \\
& =\lambda^{2}+A_{1} \lambda+A_{2}=0
\end{aligned}
$$

where $A_{1}=-(a+b)\left(z_{1}+z_{2}\right)$ and $A_{2}=\left(a b-b^{2}\right) z_{1}^{2}+\left(a^{2}-b^{2}\right) z_{1} z_{2}+\left(a b-b^{2}\right) z_{2}^{2}$.

Likewise,

$$
\operatorname{det}\left[M\left(z_{1}, z_{2}\right)-2 \lambda I\right]=4 \lambda^{2}+2 A_{1} \lambda+A_{2}=0
$$

Subtracting equation (12) from (13) gives $3 \lambda+A_{1}=0$ (because $\lambda \neq 0$ ). The condition that an eigenvalue multiplied by two is not an eigenvalue means that the polynomials $f=\lambda^{2}+$ $A_{1} \lambda+A_{2}$ and $g=3 \lambda+A_{1}$ do not have a common root. This is equivalent to the nonvanishing of the resultant:

$$
R(f, g)=\left|\begin{array}{ccc}
1 & A_{1} & A_{2} \\
3 & A_{1} & 0 \\
0 & 3 & A_{1}
\end{array}\right|=A z_{1}^{2}+2 B z_{1} z_{2}+A z_{2}^{2}
$$

This resultant is a quadratic form with $A=9\left(a b-b^{2}\right)-2(a+b)^{2}, B=\frac{1}{2}\left[9\left(a^{2}-b^{2}\right)-4(a+\right.$ $b)^{2}$. Thus a sufficient condition of 2-regularity is that this form is positively or negatively defined. Therefore one obtains $A^{2}-B^{2}>0$. In our case, one finds $A^{2}=\left[2 a^{2}-5 a b+11 b^{2}\right]^{2} \approx$ $5.49 \cdot\left(\frac{8}{\pi^{3}}\right)^{4}, B^{2}=\frac{1}{4}\left[13 b^{2}-5 a^{2}+8 b a\right]^{2} \approx 0.07 \cdot\left(\frac{8}{\pi^{3}}\right)^{4}$ and $A^{2}>B^{2}$, so the test with resultants is effective.

Summing up we conclude from the generalized Lyusternic theorem: the tangent cone coincides with the 2-kernel $\mathrm{H}_{2}(0,0)$ and this gives the solutions. 
Theorem 14 Equation (4) has a nonzero solution $u(\varepsilon)$ such that

$$
u(\varepsilon)=\varepsilon\left(\bar{z}_{1} u_{1}+\bar{z}_{2} u_{2}\right)+r(\varepsilon), \quad\|r(\varepsilon)\|=o(\varepsilon)
$$

for $\varepsilon \in(-\sigma, \sigma)$, where $\sigma>0$ is sufficiently small.

\section{Conclusion}

The paper was inspired by Buchner, Marsden and Schecter's article [8]. The authors consider the bifurcation problem $F(x, \lambda)=L x+\left(\lambda-\lambda_{0}\right) x+R(x)=0$, where $L$ is the elliptic selfadjoint operator on a suitable Banach space $Y$ of functions, with another suitable Banach space of function $X$ - the domain of $L \subset Y, R: X \rightarrow Y$ is a smooth map with $R(0)=0$ and $R^{\prime}(0)=0, \lambda_{0}$ - is an eigenvalue of $L$ of multiplicity $n, x \in X$ and $\lambda \in \mathbb{R}$. They use LyapunovSchmidt procedure to examine the above equation and show that $(0,0)$ is a bifurcation point and that there exist solutions different from $(0, \lambda)$. Our examples are special cases of the above problem, and we use $p$-regularity theory to prove the existence of solutions and give an approximative description of the solution set. The structure of the solution set is reduced to a study of the system of homogeneous algebraic equations.

\section{Competing interests}

The authors declare that they have no competing interests.

Authors' contributions

All authors read and approved the final manuscript.

\section{Author details}

${ }^{1}$ Siedlce University of Natural Sciences and Humanities, 3-go Maja 54, Siedlce, 08-110, Poland. ${ }^{2}$ System Research Institute, Polish Academy of Sciences, Newelska 6, Warsaw, 01-447, Poland. ${ }^{3}$ Dorodnicyn Computer Center, Russian Academy of

Sciences, Vavilova 40, Moscow, 119991, Russia.

\section{Acknowledgements}

This work was supported by the Russian Foundation for Basic Research Grant No. 11-01-00786-a, by the Leading Research Schools Grant No. NSH-5264.2012.1 and by the Russian Academy of Sciences Presidium Program P-18.

Received: 27 May 2013 Accepted: 28 October 2013 Published: 22 Nov 2013

\section{References}

1. Izmailov, AF, Tret'yakov, AA: Factor-Analysis of Nonlinear Mappings. Nauka, Moscow (1994) (in Russian)

2. Izmailov, AF, Tret'yakov, AA: 2-Regular Solutions of Nonlinear Problems. Theory and Numerical Methods. Nauka, Moscow (1999) (in Russian)

3. Tret'yakov, AA: Necessary conditions for optimality of $p$-th order. In: Control and Optimization, pp. 28-35. MSU, Moscow (1983) (in Russian)

4. Tret'yakov, AA: The implicit function theorem in degenerate problems. Russ. Math. Surv. 42, 179-180 (1987)

5. Prusinska, A, Tret'yakov, AA: A remark on the existence of solutions to nonlinear equations with degenerate mappings. Set-Valued Anal. 16, 93-104 (2008)

6. Brezhneva, OA, Tret'yakov, AA, Marsden, JE: Higher-order implicit function theorems and degenerate nonlinear boundary-value problems. Commun. Pure Appl. Anal. 7(2), 293-315 (2008)

7. Tret'yakov, AA, Marsden, JE: Factor analysis of nonlinear mappings: $p$-regularity theory. Commun. Pure Appl. Anal. 2(4), 425-445 (2003)

8. Buchner, M, Marsden, J, Schechter, S: Applications of the blowing-up construction and algebraic geometry to bifurcation problems. J. Differ. Equ. 48, 404-433 (1983) 